\title{
Review
}

\section{What is the role of Achromobacter species in patients with cystic fibrosis?}

\author{
Susanna Esposito ${ }^{1, *}$, Giovanna Pisi ${ }^{1}$, Valentina Fainardi ${ }^{1}$, Nicola Principi ${ }^{2}$ \\ ${ }^{1}$ Paediatric Clinic, University Hospital, Department of Medicine and Surgery, University of Parma, 43126 Parma, Italy, \\ ${ }^{2}$ Università degli Studi di Milano, 20122 Milan, Italy
}

\section{TABLE OF CONTENTS}

1. Abstract

2. Introduction

3. Achromobacter species

3.1 Characteristics

3.2 Achromobacter species sensitivity to antibiotics

4. Achromobacter species in patients with cystic fibrosis (CF)

\subsection{Frequency of detection}

4.2 Clinical relevance

5. Treatment of patients with cystic fibrosis (CF) and Achromobacter species colonization or acute exacerbation

6. Summary and perspective

7. Author contributions

8. Ethics approval and consent to participate

9. Acknowledgment

10. Funding

11. Conflict of interest

12. References

\section{Abstract}

In recent years, advances in diagnosis and treatment have significantly modified the short- and long-term prognosis of cystic fibrosis (CF) patients. However, as in the past, the most important health problem that has significantly reduced the quality of life in CF patients is the progressive deterioration of lung structure and function. In recent years, Achromobacter species have emerged with increasing incidence in the respiratory secretions of CF subjects. The significance of this detection remains debated. In this review article, the characteristics of these pathogens, the importance of their presence in CF patients, and possible antibiotic treatment of treatments for colonization and infection are discussed. Literature analysis shows that Achromobacter species, mainly A. xylosoxidans, are pathogens with intrinsic characteristics that favour persistent lung colonization and several virulence factors and secretion systems that significantly interfere with respiratory cell survival. However, although it seems undebatable that Achromobacter species detection is a marker of CF severity, the role of these pathogens as a cause of lung structure and functional deterioration is not definitively established. Nonetheless, there is general agreement about the need for antibi- otic therapy to eradicate these pathogens when they are detected in CF patients. Unfortunately, eradication is difficult, and no standard treatment is recommended by scientific societies. New possibilities are potentially offered by some recently developed drugs, such as cefiderocol, but further studies on the dosage, treatment duration and efficacy and safety of this new antibiotic in CF patients of different ages are urgently needed.

\section{Introduction}

In recent years, advances in diagnosis and treatment, including the use of transmembrane conductance regulator (CFTR) modulators for a subset of gene mutations [1], have significantly modified the short- and long-term prognosis of cystic fibrosis (CF) patients [2]. Survival has increased remarkably, and a large proportion of subjects who only 20 years ago would not have become adults can today reach over 50 years of age [3]. However, although life expectancy has improved considerably, the quality of life has been less modified. Many people with CF develop health complications. Poor nutrition status, diabetes, bile duct or intestinal obstruction, and mental health problems 
significantly complicate the lives of ageing CF patients [1]. As in the past, the most important health problem that significantly reduces the quality of life in CF patients is the progressive deterioration of lung structure and function due to chronic lung inflammation and the frequent recurrence of acute infectious episodes, more commonly referred to as pulmonary exacerbations [4].

Chronic colonization with bacterial pathogens is the main cause of progressive lung damage. Initially, during early childhood, traditional bacterial respiratory pathogens such as Haemophilus influenzae are the main colonizers. Later in life, the lung microbiology tends to progressively change. Staphylococcus aureus and Pseudomonas aeruginosa become dominant and are the main cause of all infectious respiratory clinical problems in CF patients. However, all national CF registries clearly indicate that in recent years, other microorganisms, including Achromobacter (Ac) species, have emerged and can be detected with increasing incidence in the respiratory secretions of CF subjects, highlighting the continuous modification of CF respiratory microbiota. Although the reasons for these changes are not precisely defined, it seems likely that the improved methods of bacterial identification, use of antibiotics, infection control practices, increasing prevalence of individuals with milder disease, and survivor effect may play a role in this regard $[5,6]$.

Ac species are opportunistic pathogens that have been associated with the development of severe infections, such as bacteraemia, endocarditis, pneumonia, and peritonitis [7-11]. Despite this fact, the significance of the detection of these bacteria in CF patients remains debated. It is not precisely defined whether they play a role as a cause of a more rapid deterioration of lung structure and function or they simply represent a sign of severe CF disease. In this paper, the characteristics of these pathogens, the importance of their presence in CF patients, and possible antibiotic treatment for colonization and infection will be discussed.

\section{Achromobacter species}

\subsection{Characteristics}

Ac species are gram-negative, lactose nonfermenting, catalase- and oxidase-positive bacilli that are classified as aerobic organisms, although they may also thrive in anaerobic conditions [12]. They are widely distributed in moist environments and soil and are increasingly found in hospital settings where they can be diffused through contaminated fluids. A total of 21 Ac species have been identified [12]. The most frequent isolate in CF patients worldwide is A. xylosoxidans, followed by A. ruhlandii. Other species isolates from chronic and occasional lung infection in CF patients are A. insuavis, A. deleyi, A. denitrificans, A. insolitus, A. pestifer, A. spanius and A. marplatensis.
However, as the differentiation of different species requires specific molecular-based methods, the true frequency of the various species in CF patients remains poorly defined [13]. Generally, Ac species isolated with conventional methods are reported as A. xylosoxidans [14, 15].

Similar to $P$. aeruginosa, Ac species possess a number of intrinsic characteristics that may explain both their long-term presence in the lung microbiome and their potential ability to damage lung structure and function. Their genome is highly dynamic, and hypermutation can favour adaptation of the pathogen to the lung environment and persistent colonization/infection [16]. Ac species possess a number of protein secretion systems that allow them to deliver lethal toxins into bacterial cells $[17,18]$. They are resistant to natural antimicrobial peptides contained in airway secretions [19]. Finally, they exhibit significant motility and a great ability to adhere to respiratory cells and to form biofilms, all characteristics that are important determinants of persistence, reduced sensitivity to natural defences and antibiotic activity [20, 21].

\subsection{Achromobacter species sensitivity to antibiotics}

Bacteria included in the Ac genus are generally multidrug resistant pathogens [22]. This is because they possess several intrinsic and acquired resistance mechanisms that frequently simultaneously work to make antibiotics currently used against gram-negative rods completely ineffective both in vitro and in vivo. Efflux mechanisms, beta-lactamase production and mutations in target proteins are antibiotic resistance determinants in Ac species [22]. Three different efflux mechanisms have been described. AxyABM is responsible for resistance to cephalosporins (except cefepime and cefuroxime), aztreonam, nalidixic acid, fluoroquinolones, and chloramphenicol [23]. AxyXYOprz makes aminoglycosides, cefepime, carbapenems, some fluoroquinolones, tetracyclines, and erythromycin ineffective [24, 25]. Finally, AxyEF-OprN extrudes some fluoroquinolones (levofloxacin and ciprofloxacin), tetracyclines (doxycycline and tigecycline) and carbapenems (ertapenem and imipenem) [26]. All the efflux mechanisms are intrinsic and detectable in most of the Ac species that are commonly encountered in CF patients. Only some strains that rarely colonize these subjects do not carry the AxyXYOprz efflux mechanism and remain sensitive to aminoglycosides. The production of beta-lactamases may be intrinsic (as in the case of OXA114, which makes piperacillin, ticarcillin, cephalothin and benzylpenicillin totally ineffective [27]) or acquired (such as extended-spectrum betalactamase [ESBL] and AmpC, which inactivate all betalactam antibiotics except carbapenems [28-30]). Intrinsic or acquired beta-lactamases can ultimately comprise plasmidic and chromosomal carbapenemases that generally hydrolyse all beta-lactams except aztreonam [28-30]. However, strains carrying VIM-2 beta-lactamase are also resistant to aztreonam [31-34]. 
Due to the different distributions of resistance mechanisms among Ac species and within the same species, minimum inhibitory concentration (MIC) breakpoints for these pathogens have not been definitively established. Only recently have clinical minimal inhibitory concentration breakpoints for several antibiotics been proposed by the European Committee on Antimicrobial Susceptibility Testing (EUCAST) [35]. Moreover, sensitivity to antibiotics can significantly vary from strain to strain and seems to gradually decrease, probably because of within-host bacterial genome evolution during chronic colonization [36]. This can explain why studies carried out in different countries and in different periods of time have reported different results. The global evaluation of nine case series published from 2003 to 2014 showed that the most effective in vitro antibiotics were ticarcillin (99.5\%), cefoperazonesulbactam (98.7\%), piperacillin-tazobactam (97.2\%), and imipenem (86.4\%), whereas aztreonam and tetracyclines displayed poor sensitivity (1.5\% and $17.5 \%$, respectively) [37]. In a study carried out in Argentina in which 59 strains of Ac species were collected from clinical specimens of patients, similar to what had been previously reported by other authors [38, 39], the most active antibiotics were piperacillin alone or with tazobactam and carbapenems, with meropenem significantly more effective than imipenem and ertapenem [40]. Co-trimoxazole, minocycline, and colistin were at least partially active. Among cephalosporins, ceftazidime was more effective than cefepime [40]. In UK, in a sample of 112 Ac spp. from CF patients, piperacillin-tazobactam (70.2\%) and cotrimoxazole (69.7\%) were the most active antibiotics [41]. Finally, in France, colistin was found to be effective in 19 out of 22 Ac strains collected from CF patients [42].

Combinations of antibiotics were found to exert a synergistic interaction that in some cases significantly increased the bactericidal activity of the single agent, providing evidence for potentially effective in vivo therapies. A very good example in this regard has been reported by Daman-Çelik et al. [43]. These authors tested meropenem alone or in combination with colistin, levofloxacin, and chloramphenicol and found that when meropenem was combined with colistin, the combination was effective against bacteria susceptible to meropenem and colistin but also against colistin resistance. In contrast, the meropenemlevofloxacin combination had a synergistic but not bactericidal effect, whereas the meropenem-chloramphenicol combination was neither synergistic nor bactericidal [43].

\section{Achromobacter species in patients with cystic fibrosis (CF)}

\subsection{Frequency of detection}

In the last 20 years, Ac species have been detected with increased frequency in the respiratory secretions of CF patients [44]. Several factors could explain the emergence of these pathogens $[45,45]$. The use of aggressive antibiotic therapy in an attempt to eliminate typical CF pathogens, such as $S$. aureus and $P$. aeruginosa, may have strongly induced bacterial selection. Evidence of Ac species may also have been favoured by the introduction in clinical practice of advanced bacterial detection methods, more careful CF patient follow-up, and prolongation of the average lifespan. Finally, it cannot be excluded that the increased detection may be ascribed to some Ac species characteristics, mainly constitutive resistance to many antibiotics and the ability to adapt to surrounding pressures by means of within-host genome evolution, all factors that may favour persistence in respiratory secretions [44-46].

Despite detection in almost all studies, the incidence of Ac species identification in CF patients varies significantly from study to study, ranging from 3\% to $30 \%$ [47-49]. The criteria used to define colonization (sporadic, intermittent, or chronic) and the time of evaluation can explain the differences. The lowest values were associated with chronic colonization and with collection of respiratory samples in the first years of this century [50]. A study carried out in France clearly showed that the detection of Ac species in respiratory secretions of CF patients increased from 1999 to 2018 from $3.1 \%$ to $6.7 \%$ [51]. Similar data have been collected in the USA [52].

\subsection{Clinical relevance}

The clinical relevance of Ac species in CF patients remains debated. Several retrospective studies have shown that CF patients with severe disease are more frequently infected by $A$. xylosoxidans than are patients with less severe signs and symptoms of lung involvement [5355]. Worse lung function, more frequent pulmonary exacerbations and the need for hospitalization and antibiotic treatment were more common among $A$. xylosoxidans-infected patients than among noninfected controls [53-55]. This explains why the detection of this pathogen in a CF patient is considered a marker of severe CF. However, as few studies for species other than A. xylosoxidans exist, the greater clinical relevance of this pathogen is not definitively established. On the other hand, the role of Ac species infection as a cause of primary deterioration of lung function is far from definitively ascertained. Support for this hypothesis is given by the evidence that Ac species can cause a significant inflammatory status similar to that caused by P. aeruginosa and are potentially able to cause progressive lung damage per se. Hansen et al. measured cytokine levels in the serum and sputum of 11 healthy controls and 60 CF patients, 11 with $A$. xylosoxidans, 11 with the $B$. cepacia complex, 21 with $P$. aeruginosa and 17 without infection with these pathogens [56]. They found that all of the chronically infected patients had significantly higher serum levels of interferon (IFN)- $\gamma$ and interleukin (IL)-6 than noninfected CF patients. However, only A. xylosoxidans and P. aeruginosa 
patients had significantly higher sputum tumour necrosis factor (TNF)- $\alpha$ and serum granulocyte colony stimulating factor (G-CSF) levels, suggesting that these bacteria could cause greater lung damage.

Clinical findings do not definitively solve the problem of the causative role of Ac species. Indeed, data collected in several studies have not answered this question. In one investigation, respiratory function and exacerbation frequency were compared in CF patients with at least one sputum culture positive for $A$. xylosoxidans and in control uninfected patients [57]. Data collected between 1 year prior to and 3 years after $A$. xylosoxidans isolation were considered. Compared to negative patients, positive subjects showed a greater decline in forced expiratory volume in 1 second (FEV1) in the first year $\left(-153.6 \pm 16.1 \mathrm{~mL} \cdot\right.$ year $^{-1}$ vs. $\left.-63.8 \pm 18.5 \mathrm{~mL} \cdot \mathrm{year}^{-1} ; p=0.0003\right)$, together with more exacerbations in the first 3 years after pathogen detection ( 9 vs. $7 ; p=0.03$ ). However, these findings do not definitively demonstrate a causal relationship between the presence of the pathogen and the worsening of pulmonary disease because the subjects with $A$. xylosoxidans had, at the beginning of the study, a worse clinical situation and greater FEV1 decline, and more exacerbations were mainly evidenced in A. xylosoxidans cases co-colonized with $P$ aeruginosa (75\%) [56]. Similar inconclusive findings were reported by Edwards et al. [58], Firmida et al. [59], Somayaji et al. [55], and Marsac et al. [60]. The first authors found that if CF patients were more likely to experience pulmonary exacerbation with incident Ac species-positive cultures (42\% vs. 21\%; odds ratio [OR], 2.7; 95\% confidence interval [CI], 1.1-6.7; $p=0.03$ ), persistent infection was associated with neither annual lung function decline ( $-1.08 \%$ [95\% CI, -2.73 to $0.57 \%$ ] vs. $-2.74 \%$ [95\% CI, -4.02 to $1.46 \%$ ]; $p=0.12$ ) nor the risk of pulmonary exacerbations (OR, 1.21 [95\% CI, 0.45 to 3.28]; $p=0.70$ ) [55]. In a case-control retrospective study, the clinical course of a group of chronically or intermittently A. xylosoxidanscolonized/infected CF patients was compared with that of never colonized/infected subjects during two periods that were 2 years apart [61]. No differences in lung function among groups over 2 years were evidenced, although in the chronically colonized/infected subjects, a trend towards a greater decrease in lung function was observed $\mathbf{5 1 . 7 \%}$ in the chronic colonization/infection group vs. $82.7 \%$ in the intermittent colonization/infection group vs. $76 \%$ in the never colonized/infected group) [55]. Somayaji et al. [55] studied 88 patients who had one or more cultures positive for Ac species during the course of 18 years. They found that pulmonary exacerbations and risk of death or transplantation were more common in these subjects than in uninfected CF patients. However, further evaluations did not show any independent association between chronic Ac species infection and worsening of the risk of pulmonary exacerbation, lung function deterioration or the time lag for death or lung transplantation. Finally, a recent case control study by Marsac et al. carried out in two French paediatric centres compared 45 patients infected by A. xylosoxidans with the same number of never infected controls matched for age, sex, pancreatic status and genotype [60]. Clinical data collected in the two years immediately preceding and following the first identification of the pathogen were evaluated. CF severity was significantly greater in $A$. xylosoxidans patients than in controls both before and after pathogen detection. Pulmonary exacerbations, hospitalization, and the need for antibiotic courses were significantly more frequent in positive than in negative patients. Moreover, lung function decline tended to be faster in cases ($5.5 \%$ vs. $-0.5 \%$ per year). However, even in this study, the greater colonization with Pseudomonas aeruginosa in A. xylosoxidans-positive patients $(p=0.0002)$ makes these findings difficult to interpret [60].

\section{Treatment of patients with cystic fibrosis (CF) and Achromobacter species colonization or acute exacerbation}

Similar to what has been reported for Pseudomonas aeruginosa [61] and Burkholderia cepacia complex [62], for Ac species, transmission of these pathogens between CF patients has also been demonstrated [63]. This means that to avoid Ac species infection, close contact between chronically infected patients and noninfected patients should be avoided. No standard treatment for Ac species eradication in CF patients is presently recommended by scientific societies. Several other factors besides the already cited differences in sensitivity to antibiotics among Ac species can explain this limitation. The total number of patients with Ac species infection enrolled in studies evaluating the efficacy of antibiotic therapy in CF patients is too small to draw definitive conclusions. Coinfection with other pathogens, such as $P$. aeruginosa, can significantly hamper antibiotic treatment efficacy evaluations. The role of the addition of inhaled antibiotics to standard systemic therapy in Ac species eradication is not definitively established, although a study has shown that $56 \%$ of patients who received inhalation therapy with ceftazidime, colistin, or tobramycin were not colonized by Ac species after three years, compared to $13 \%$ of patients who were not given inhaled antibiotics [64]. All these findings seem to suggest that treatment of Ac species infection in CF patients should be evaluated on a case-by-case basis, considering the patient medical history, frequency of respiratory exacerbations, infection severity, antibiotics previously administered, and in vitro antibiotic susceptibility of previous and current infecting bacteria. However, while waiting for a microbiological response, as generally suggested for pulmonary exacerbations in CF patients with possible multiresistant pathogens, prescription of a combination therapy including carbapenems is considered the best solution $[65,66]$. Some suggestions for initial therapy can 
also be drawn from some studies carried out in patients experiencing Ac species infections cited outside the context of CF [67]. In a group of 34 trauma patients with a total of 37 episodes of Ac species-related ventilatory associated pneumonia, clinical success was achieved in $87 \%$ of the cases using imipenem/cilastatin, cefepime, or cotrimoxazole [68]. Moreover, most of the patients with A. $x y$ losoxidans bacteraemia receiving ceftazidime, piperacillin, ticarcillin and cotrimoxazole given alone or in combination had a positive evolution [69]. Finally, ceftazidime was effective in patients with $A$. xylosoxidans meningitis [70]. However, it must be highlighted that the results of that study remain debatable as, together with antimicrobial drugs, other therapeutic interventions that may have played a role in favouring infection eradication were simultaneously put in place. In bacteraemic patients and in subjects with meningitis, central venous catheters and epidural catheters were removed $[69,70]$. On the other hand, administration of piperacillin-tazobactam, meropenem, and imipenem monotherapies to a group of elderly people with hospital-acquired pneumonia due to Ac spp. was only partially effective, as 5 out of 15 patients died on Day 30 of treatment [71].

A valuable contribution to the effective treatment of Ac species infection may be offered by cefiderocol. This is a new-generation parenteral siderophore cephalosporin that, as with other beta-lactam antibiotics, acts by inhibiting bacterial cell wall synthesis [72]. However, it has increased bacterial killing properties, as it exhibits improved stability to beta-lactamases and is actively taken up by gram-negative bacteria under iron-depleted conditions [73]. This drug has been found to be effective in vitro against a great number of gram-negative rods, including multidrugresistant Enterobacteriaceae and nonfermenting organisms [74]. Data regarding Ac species are limited, but some evaluations have reported very low minimum inhibitory concentrations, suggesting potentially high therapeutic activity in severe infections due to this pathogen [74]. Cefiderocol is approved by the Food and Drug Administration (FDA) for the treatment of complicated urinary tract infections, hospital-acquired bacterial pneumonia and ventilatorassociated bacterial pneumonia in adults when these diseases are caused by gram-negative bacteria resistant to other antibiotics. It is administered at a dosage of 2 grams over a three-hour period every eight hours for 7-14 days [75]. However, no efficacy data in CF and paediatric patients are available, and the dosage in CF patients of any age has not been established. Compassionate use of the drug was conducted in 8 adult and paediatric patients with A. xylosoxidans infection who received 12 courses of the drug together, in 11 cases with other antibiotics effective against gramnegative rods [76]. The duration of cefiderocol administration varied from 2 days to 6 weeks. Resolution or improvement of disease symptoms after 30 days was observed in 11 cases, although in 3 patients, pretreatment evaluation revealed in vitro pathogen resistance. Unfortunately, microbiologic relapse was observed after 11 of 12 treatment courses, notably without emergence of resistance. Further evidence of the potential in vivo efficacy of cefiderocol in CF patients infected by Ac species is given by the case of a 10-year-old female treated with this antibiotic along with meropenem/vaborbactam and bacteriophage therapy [77]. Despite in vitro resistance of the pathogen to both cefiderocol and meropenem/vaborbactam, the patient's lung function improved dramatically, and the pathogen was eradicated from respiratory secretions 8 and 16 weeks after completion of therapy [77]. Finally, potential efficacy against Ac species infection is ascribed [78] to the meropenemvaborbactam combination [79] and to eravacycline [80] for their in vitro activity against a number of gram-negative rods. However, for both preparations, no efficacy in CF and no dosage for CF patients are presently available.

\section{Summary and perspective}

Ac species, mainly A. xylosoxidans, are pathogens that have only recently been associated with CF. However, they have intrinsic characteristics that favour persistent lung colonization and several virulence factors and secretion systems that significantly interfere with respiratory cell survival. Finally, the bacteria can adhere to and penetrate respiratory cells and form biofilms that significantly reduce the host ability to combat them.

In many aspects, Ac species resemble $P$. aeruginosa. These findings and the evidence that Ac species are generally detected in patients with severe CF make them a significant component of CF respiratory microbiota. However, although it seems undebatable that Ac species detection is a marker of CF severity, the role of these pathogens as a cause of lung structure and function deterioration is not definitively established. Further studies are needed to solve this problem. Nonetheless, there is general agreement about the need for antibiotic therapy to eradicate these pathogens when they are detected in CF patients. Unfortunately, eradication is difficult, and no standard treatment is recommended by scientific societies. Antibiotics generally used in the treatment of gram-negative multiresistant strains are suggested. New possibilities are potentially offered by some recently developed drugs, such as cefiderocol, but further studies on the dosage, treatment duration and efficacy and safety of this new antibiotic in CF patients of different ages are urgently needed.

\section{Author contributions}

SE co-wrote the first draft of the manuscript; GP and VF performed the literature review and gave a substantial scientific contribution. NP co-wrote the manuscript and gave a substantial scientific contribution. All the authors approved the final version of the manuscript. 


\section{Ethics approval and consent to participate}

Not applicable.

\section{Acknowledgment}

Not applicable.

\section{Funding}

The publication of this review was funded from the Cystic Fibrosis Center of Parma, University Hospital, Parma, Italy.

\section{Conflict of interest}

The authors declare no conflict of interest.

\section{References}

[1] Spanò V, Venturini A, Genovese M, Barreca M, Raimondi MV, Montalbano A, et al. Current development of CFTR potentiators in the last decade. European Journal of Medicinal Chemistry. 2020; 204: 112631

[2] Rey MM, Bonk MP, Hadjiliadis D. Cystic Fibrosis: Emerging Understanding and Therapies. Annual Review of Medicine. 2019; 70: 197-210.

[3] McKone EF, Ariti C, Jackson A, Zolin A, Carr SB, Orenti A, et al. Survival estimates in European cystic fibrosis patients and the impact of socioeconomic factors: a retrospective registry Cohort study. European Respiratory Journal. 2021. (in press)

[4] Wendekier C, Wendekier-Raybuck K. Cystic fibrosis: a changing landscape. Nursing. 2021; 51: 32-38.

[5] Parkins MD, Floto RA. Emerging bacterial pathogens and changing concepts of bacterial pathogenesis in cystic fibrosis. Journal of Cystic Fibrosis. 2015; 14: 293-304.

[6] Cystic Fibrosis Foundation. 2019 Patient Registry. Annual Data Report. Available at: https://www.cff.org/Research/Researche r-Resources/Patient-Registry/2019-Patient-Registry-Annual-D ata-Report.pdf (Accessed: 24 July 2021).

[7] Marion-Sanchez K, Pailla K, Olive C, Le Coutour X, Derancourt C. Achromobacter spp. healthcare associated infections in the French West Indies: a longitudinal study from 2006 to 2016. BMC Infectious Diseases. 2019; 19: 795.

[8] D’Amato RF, Salemi M, Mathews A, Cleri DJ, Reddy G. Achromobacter xylosoxidans (Alcaligenes xylosoxidans subsp. xylosoxidans) meningitis associated with a gunshot wound. Journal of Clinical Microbiology. 1988; 26: 2425-2426.

[9] Tena D, González-Praetorius A, Pérez-Balsalobre M, Sancho O, Bisquert J. Urinary tract infection due to Achromobacter xylosoxidans: report of 9 cases. Scandinavian Journal of Infectious Diseases. 2008; 40: 84-87.

[10] Tena D, Martínez NM, Losa C, Solís S. Skin and soft tissue infection caused by Achromobacter xylosoxidans: report of 14 cases. Scandinavian Journal of Infectious Diseases. 2014; 46: 130-135.

[11] Asano K, Tada S, Matsumoto T, Miyase S, Kamio T, Sakurai K, et al. A novel bacterium Achromobacter xylosoxidans as a cause of liver abscess: three case reports. Journal of Hepatology. 2005; 43: 362-365.

[12] Spilker T, Vandamme P, Lipuma JJ. Identification and distribution of Achromobacter species in cystic fibrosis. Journal of Cystic Fibrosis. 2013; 12: 298-301.
[13] Price EP, Soler Arango V, Kidd TJ, Fraser TA, Nguyen TK, Bell SC, et al. Duplex real-time PCR assay for the simultaneous detection of Achromobacter xylosoxidans and Achromobacter spp. Microbial Genomics. 2020; 6: mgen000406.

[14] Ridderberg W, Wang M, Nørskov-Lauritsen N. Multilocus sequence analysis of isolates of Achromobacter from patients with cystic fibrosis reveals infecting species other than Achromobacter xylosoxidans. Journal of Clinical Microbiology. 2012; 50: 2688-2694.

[15] Cools P, Ho E, Vranckx K, Schelstraete P, Wurth B, Franckx $\mathrm{H}$, et al. Epidemic Achromobacter xylosoxidans strain among Belgian cystic fibrosis patients and review of literature. BMC Microbiology. 2016; 16: 122.

[16] Veschetti L, Sandri A, Johansen HK, Lleò MM, Malerba G. Hypermutation as an evolutionary mechanism for Achromobacter xylosoxidans in cystic fibrosis lung infection. Pathogens. 2020; 9: 72.

[17] Hood RD, Singh P, Hsu F, Güvener T, Carl MA, Trinidad RRS, et al. A type VI secretion system of Pseudomonas aeruginosa targets a toxin to bacteria. Cell Host \& Microbe. 2010; 7: 2537.

[18] Sgro GG, Oka GU, Souza DP, Cenens W, Bayer-Santos E, Matsuyama BY, et al. Bacteria-Killing Type IV Secretion Systems. Frontiers in Microbiology. 2019; 10: 1078.

[19] Cabak A, Hovold G, Petersson A, Ramstedt M, Påhlman LI. Activity of airway antimicrobial peptides against cystic fibrosis pathogens. Pathogens and Disease. 2020; 78: ftaa048

[20] Di Bonaventura G, Prosseda G, Del Chierico F, Cannavacciuolo S, Cipriani P, Petrucca A, et al. Molecular characterization of virulence determinants of Stenotrophomonas maltophilia strains isolated from patients affected by cystic fibrosis. International Journal of Immunopathology and Pharmacology. 2007; 20: 529-537.

[21] Pompilio A, Crocetta V, Confalone P, Nicoletti M, Petrucca A, Guarnieri S, et al. Adhesion to and biofilm formation on IB3-1 bronchial cells by Stenotrophomonas maltophilia isolates from cystic fibrosis patients. BMC Microbiology. 2010; 10: 102.

[22] Menetrey Q, Sorlin P, Jumas-Bilak E, Chiron R, Dupont C, Marchandin H. Achromobacter xylosoxidans and Stenotrophomonas maltophilia: Emerging Pathogens WellArmed for Life in the Cystic Fibrosis Patients' Lung. Genes. 2021; 12: 610.

[23] Bador J, Amoureux L, Duez J, Drabowicz A, Siebor E, Llanes C, et al. First description of an RND-type multidrug efflux pump in Achromobacter xylosoxidans, AxyABM. Antimicrobial Agents and Chemotherapy. 2011; 55: 4912-4914.

[24] Bador J, Amoureux L, Blanc E, Neuwirth C. Innate aminoglycoside resistance of Achromobacter xylosoxidans is due to AxyXY-OprZ, an RND-type multidrug efflux pump. Antimicrobial Agents and Chemotherapy. 2013; 57: 603-605.

[25] Hu Y, Zhu Y, Ma Y, Liu F, Lu N, Yang X, et al. Genomic insights into intrinsic and acquired drug resistance mechanisms in Achromobacter xylosoxidans. Antimicrobial Agents and Chemotherapy. 2015; 59: 1152-1161.

[26] Nielsen SM, Penstoft LN, Nørskov-Lauritsen N. Motility, Biofilm Formation and Antimicrobial Efflux of Sessile and Planktonic Cells of Achromobacter xylosoxidans. Pathogens. 2019; 8: 14.

[27] Doi Y, Poirel L, Paterson DL, Nordmann P. Characterization of a naturally occurring class $D$ beta-lactamase from Achromobacter xylosoxidans. Antimicrobial Agents and Chemotherapy. 2008; 52: 1952-1956.

[28] Neuwirth C, Freby C, Ogier-Desserrey A, Perez-Martin S, Houzel A, Péchinot A, et al. VEB-1 in Achromobacter xylosoxidans from cystic fibrosis patient, France. Emerging Infectious Diseases. 2006; 12: 1737-1739.

[29] Traglia GM, Almuzara M, Merkier AK, Adams C, Galanternik L, Vay C, et al. Achromobacter xylosoxidans: an Emerging 
Pathogen Carrying Different Elements Involved in Horizontal Genetic Transfer. Current Microbiology. 2012; 65: 673-678.

[30] Vali P, Shahcheraghi F, Seyfipour M, Zamani MA, Allahyar MR, Feizabadi MM. Phenotypic and Genetic Characterization of Carbapenemase and ESBLs Producing Gram-negative Bacteria (GNB) Isolated from Patients with Cystic Fibrosis (CF) in Tehran Hospitals. Journal of Clinical and Diagnostic Research. 2014; 8: 26-30.

[31] Riccio ML, Pallecchi L, Fontana R, Rossolini GM. In70 of plasmid pAX22, a bla(VIM-1)-containing integron carrying a new aminoglycoside phosphotransferase gene cassette. Antimicrobial Agents and Chemotherapy. 2001; 45: 1249-1253.

[32] Shin KS, Han K, Lee J, Hong SB, Son BR, Youn SJ, et al. Imipenem-resistant Achromobacter xylosoxidans carrying blaVIM-2-containing class 1 integron. Diagnostic Microbiology and Infectious Disease. 2005; 53: 215-220.

[33] Sofianou D, Markogiannakis A, Metzidie E, Pournaras S, Tsakris A. VIM-2 metallo- $\beta$-lactamase in Achromobacter xylosoxidans in Europe. European Journal of Clinical Microbiology \& Infectious Diseases. 2005; 24: 854-855.

[34] El Salabi A, Borra PS, Toleman MA, Samuelsen Ø, Walsh TR. Genetic and biochemical characterization of a novel metallo- $\beta$ lactamase, TMB-1, from an Achromobacter xylosoxidans strain isolated in Tripoli, Libya. Antimicrobial Agents and Chemotherapy. 2012; 56: 2241-2245.

[35] European Committee on Antimicrobial Susceptibility Testing (EUCAST). Achromobacter xylosoxidans - proposed method and breakpoints. Consultation closed 31 October, 2020. Comments and EUCAST response. 2020. Available at: https://ww w.eucast.org/publications_and_documents/consultations/ (Accesswd: 6 September 2021).

[36] Amoureux L, Bador J, Siebor E, Taillefumier N, Fanton A, Neuwirth C. Epidemiology and resistance of Achromobacter xylosoxidans from cystic fibrosis patients in Dijon, Burgundy: first French data. Journal of Cystic Fibrosis. 2013; 12: 170-176.

[37] Swenson CE, Sadikot RT. Achromobacter respiratory infections. Annals of the American Thoracic Society. 2015; 12: 252-258.

[38] Chandrasekar PH, Arathoon E, Levine DP. Infections due to Achromobacter xylosoxidans. Case report and review of the literature. Infection. 1986; 14: 279-282.

[39] Fass RJ, Barnishan J. In vitro susceptibilities of nonfermentative gram-negative bacilli other than Pseudomonas aeruginosa to 32 antimicrobial agents. Reviews of Infectious Diseases. 1980; 2: 841-853.

[40] Almuzara M, Limansky A, Ballerini V, Galanternik L, Famiglietti A, Vay C. In vitro susceptibility of Achromobacter spp. isolates: comparison of disk diffusion, Etest and agar dilution methods. International Journal of Antimicrobial Agents. 2010; 35: 68-71.

[41] Okoliegbe IN, Hijazi K, Cooper K, Ironside C, Gould IM. Longitudinal Surveillance and Combination Antimicrobial Susceptibility Testing of Multidrug-Resistant Achromobacter Species from Cystic Fibrosis Patients. Antimicrobial Agents and Chemotherapy. 2020; 64: e01467-20.

[42] Biswas S, Dubus J, Reynaud-Gaubert M, Stremler N, Rolain J. Evaluation of colistin susceptibility in multidrug-resistant clinical isolates from cystic fibrosis, France. European Journal of Clinical Microbiology \& Infectious Diseases. 2013; 32: 14611464.

[43] Damar-Çelik D, Nørskov-Lauritsen N, Özbek-Çelik B. Comparative in vitro activities of meropenem in combination with colistin, levofloxacin, or chloramphenicol against Achromobacter xylosoxidans strains isolated from patients with cystic fibrosis. Journal of Global Antimicrobial Resistance. 2020; 22: 713-717.

[44] Ridderberg W, Nielsen SM, Nørskov-Lauritsen N. Genetic Adaptation of Achromobacter sp. during Persistence in the Lungs of Cystic Fibrosis Patients. PLoS ONE. 2015; 10: e0136790.

[45] Ormerod KL, George NM, Fraser JA, Wainwright C, Hugen- holtz P. Comparative genomics of non-pseudomonal bacterial species colonising paediatric cystic fibrosis patients. PeerJ. 2015; 3: e1223.

[46] Jeukens J, Freschi L, Vincent AT, Emond-Rheault J, KukavicaIbrulj I, Charette SJ, et al. A Pan-Genomic Approach to Understand the Basis of Host Adaptation in Achromobacter. Genome Biology and Evolution. 2017; 9: 1030-1046.

[47] De Baets F, Schelstraete P, Van Daele S, Haerynck F, Vaneechoutte M. Achromobacter xylosoxidans in cystic fibrosis: prevalence and clinical relevance. Journal of Cystic Fibrosis. 2007; 6: 75-78.

[48] Raso T, Bianco O, Grosso B, Zucca M, Savoia D. Achromobacter xylosoxidans respiratory tract infections in cystic fibrosis patients. Acta Pathologica, Microbiologica, Et Immunologica Scandinavica. 2008; 116: 837-841.

[49] Spicuzza L, Sciuto C, Vitaliti G, Di Dio G, Leonardi S, La Rosa $M$. Emerging pathogens in cystic fibrosis: ten years of follow-up in a cohort of patients. European Journal of Clinical Microbiology \& Infectious Diseases. 2009; 28: 191-195.

[50] Gade SS, Nørskov-Lauritsen N, Ridderberg W. Prevalence and species distribution of Achromobacter sp. cultured from cystic fibrosis patients attending the Aarhus centre in Denmark. Journal of Medical Microbiology. 2017; 66: 686-689.

[51] Menetrey Q, Dupont C, Chiron R, Marchandin H. Bactéries émergentes dans la mucoviscidose et les dilatations des bronches hors mucoviscidose. Le point de vue du microbiologiste. Revue Des Maladies Respiratoires. 2020; 37: 561-571.

[52] Cystic Fibrosis Foundation Patient Registry 2019 Annual Data Report. Available at: https://www.cff.org/Research/Researche r-Resources/Patient-Registry/2019-Patient-Registry-Annual-D ata-Report.pdf (Accessed: 29 July 2021).

[53] Recio R, Branas P, Martinez MT, Chaves F, Orellana MA. Effect of respiratory Achromobacter spp. infection on pulmonary function in patients with cystic fibrosis. Journal of Medical Microbiology. 2018; 67: 952-956.

[54] Tetart M, Wallet F, Kyheng M, Leroy S, Perez T, Le Rouzic O, et al. Impact of Achromobacter xylosoxidans isolation on the respiratory function of adult patients with cystic fibrosis. ERJ Open Research. 2019; 5: 00051-2019.

[55] Somayaji R, Stanojevic S, Tullis DE, Stephenson AL, Ratjen F, Waters V. Clinical Outcomes Associated with Achromobacter Species Infection in Patients with Cystic Fibrosis. Annals of the American Thoracic Society. 2017; 14: 1412-1418.

[56] Hansen CR, Pressler T, Nielsen KG, Jensen PØ, Bjarnsholt T, Høiby N. Inflammation in Achromobacter xylosoxidans infected cystic fibrosis patients. Journal of Cystic Fibrosis. 2010; 9: 51-58.

[57] Gibson RL, Burns JL, Ramsey BW. Pathophysiology and management of pulmonary infections in cystic fibrosis. American Journal of Respiratory and Critical Care Medicine. 2003; 168: 918-951.

[58] Edwards BD, Greysson-Wong J, Somayaji R, Waddell B, Whelan FJ, Storey DG, et al. Prevalence and Outcomes of Achromobacter Species Infections in Adults with Cystic Fibrosis: a North American Cohort Study. Journal of Clinical Microbiology. 2017; 55: 2074-2085.

[59] Firmida MC, Pereira RHV, Silva EASR, Marques EA, Lopes AJ. Clinical impact of Achromobacter xylosoxidans colonization/infection in patients with cystic fibrosis. Brazilian Journal of Medical and Biological Research. 2016; 49: e5097.

[60] Marsac C, Berdah L, Thouvenin G, Sermet-Gaudelus I, Corvol $\mathrm{H}$. Achromobacter xylosoxidans airway infection is associated with lung disease severity in children with cystic fibrosis. ERJ Open Research. 2021; 7: 00076-02021.

[61] Ojeniyi B, Frederiksen B, Hoiby N. Pseudomonas aeruginosa cross-infection among patients with cystic fibrosis during a winter camp. Pediatric Pulmonology. 2000; 29: 177-181.

[62] Burns JL. Burkholderia cepacia-a transmissible cystic fibrosis pathogen. The Journal of Pediatrics. 2001; 139: 618-619. 
[63] Raso T, Bianco O, Grosso B, Zucca M, Savoia D. Achromobacter xylosoxidans respiratory tract infections in cystic fibrosis patients. Acta Pathologica, Microbiologica, et Immunologica Scandinavica. 2008; 116: 837-841.

[64] Wang M, Ridderberg W, Hansen CR, Høiby N, Jensen-Fangel S, Olesen HV, et al. Early treatment with inhaled antibiotics postpones next occurrence of Achromobacter in cystic fibrosis. Journal of Cystic Fibrosis. 2013; 12: 638-643.

[65] Bilton D, Canny G, Conway S, Dumcius S, Hjelte L, Proesmans $\mathrm{M}$, et al. Pulmonary exacerbation: towards a definition for use in clinical trials. Report from the EuroCareCF Working Group on outcome parameters in clinical trials. Journal of Cystic Fibrosis. 2011; 10: S79-S81.

[66] Smyth AR, Bell SC, Bojcin S, Bryon M, Duff A, Flume P, et al. European Cystic Fibrosis Society Standards of Care: Best Practice guidelines. Journal of Cystic Fibrosis. 2014; 13 Suppl 1: S23-S42.

[67] Isler B, Kidd TJ, Stewart AG, Harris P, Paterson DL. Achromobacter Infections and Treatment Options. Antimicrobial Agents and Chemotherapy. 2020; 64: e01025-20.

[68] Wood GC, Jonap BL, Maish GO, Magnotti LJ, Swanson JM, Boucher BA, et al. Treatment of Achromobacter VentilatorAssociated Pneumonia in Critically Ill Trauma Patients. The Annals of Pharmacotherapy. 2018; 52: 120-125.

[69] Legrand C, Anaissie E. Bacteremia due to Achromobacter xylosoxidans in patients with cancer. Clinical Infectious Diseases. 1992; 14: 479-484.

[70] Ramos JM, Fernández-Roblas R, García-Ruiz P, Soriano F. Meningitis caused by Alcaligenes (Achromobacter) xylosoxidans associated with epidural catheter. Infection. 1995; 23: 395396.

[71] Liu C, Pan F, Guo J, Yan W, Jin Y, Liu C, et al. Hospital Acquired Pneumonia Due to Achromobacter spp. in a Geriatric Ward in China: Clinical Characteristic, Genome Variability, Biofilm Production, Antibiotic Resistance and Integron in Isolated Strains. Frontiers in Microbiology. 2016; 7: 621.

[72] Zhanel GG, Golden AR, Zelenitsky S, Wiebe K, Lawrence CK, Adam HJ, et al. Cefiderocol: a Siderophore Cephalosporin with Activity against Carbapenem-Resistant and Multidrug-Resistant Gram-Negative Bacilli. Drugs. 2019; 79: 271-289.

[73] Abdul Mutakabbir JC, Alosaimy S, Morrisette T, Kebriaei R, Rybak MJ. Cefiderocol: a Novel Siderophore Cephalosporin against Multidrug Resistant Gram Negative Pathogens. Pharmacotherapy. 2020; 40: 1228-1247.
[74] Rolston KVI, Gerges B, Raad I, Aitken SL, Reitzel R, Prince R. 1375. in vitro Activity of Cefiderocol and Comparator Agents against Gram-Negative Isolates from Cancer Patients. Open Forum Infectious Diseases. 2018; 5: S421-S422.

[75] Gavioli EM, Guardado N, Haniff F, Deiab N, Vider E. Does Cefiderocol Have a Potential Role in Cystic Fibrosis Pulmonary Exacerbation Management? Microbial Drug Resistance. 2021. (in press)

[76] Warner NC, Bartelt LA, Lachiewicz AM, Tompkins KM, Miller MB, Alby K, et al. Cefiderocol for the Treatment of Adult and Pediatric Patients with Cystic Fibrosis and Achromobacter xylosoxidans Infections. Clinical Infectious Diseases. 2020. (in press)

[77] Gainey AB, Burch AK, Brownstein MJ, Brown DE, Fackler J, Horne B, et al. Combining bacteriophages with cefiderocol and meropenem/vaborbactam to treat a pan-drug resistant Achromobacter species infection in a pediatric cystic fibrosis patient. Pediatric Pulmonology. 2020; 55: 2990-2994.

[78] Belcher R, Zobell JT. Optimization of antibiotics for cystic fibrosis pulmonary exacerbations due to highly resistant nonlactose fermenting Gram negative bacilli: Meropenem vaborbactam and cefiderocol. Pediatric Pulmonology. 2021; 56: 3059-3061.

[79] Caverly LJ, Spilker T, Kalikin LM, Stillwell T, Young C, Huang $\mathrm{DB}$, et al. In Vitro Activities of $\beta$-Lactam- $\beta$-Lactamase Inhibitor Antimicrobial Agents against Cystic Fibrosis Respiratory Pathogens. Antimicrob Agents Chemother. 2019; 64: e0159519.

[80] Canonica T, Thompson S, Carr A, Alexander J, Minor SB. 2275 Novel therapeutic options for the treatment of multi-drugresistant Achromobacter respiratory infections. Open Forum Infectious Diseases, 2019; 6: S779-S779.

Keywords: Achromobacter species; Cefiderocol; Cystic fibrosis; Multidrug-resistant bacteria; Pseudomonas aeruginosa; Respiratory exacerbation

Send correspondence to: Susanna Esposito, Paediatric Clinic, University Hospital, Department of Medicine and Surgery, University of Parma, 43126 Parma, Italy, E-mail: susannamariaroberta.esposito@unipr.it 